\title{
Multi-Granulation Neutrosophic Rough Sets on a Single Domain and Dual Domains with Applications
}

\author{
Chunxin Bo ${ }^{1}$, Xiaohong Zhang ${ }^{2,3, *(1)}$, Songtao Shao ${ }^{1}$ and Florentin Smarandache ${ }^{4}$ (i) \\ 1 College of Information Engineering, Shanghai Maritime University, Shanghai 201306, China; \\ 201640311001@stu.shmtu.edu.cn (C.B.); 201740310005@stu.shmtu.edu.cn (S.S.) \\ 2 Department of Mathematics, Shaanxi University of Science \& Technology, Xi'an 710021, China \\ 3 Department of Mathematics, Shanghai Maritime University, Shanghai 201306, China \\ 4 Department of Mathematics, University of New Mexico, Gallup, NM 87301, USA; smarand@unm.edu \\ * Correspondence: zhangxiaohong@sust.edu.cn or zhangxh@shmtu.edu.cn
}

Received: 28 June 2018; Accepted: 18 July 2018; Published: 23 July 2018

\begin{abstract}
It is an interesting direction to study rough sets from a multi-granularity perspective. In rough set theory, the multi-particle structure was represented by a binary relation. This paper considers a new neutrosophic rough set model, multi-granulation neutrosophic rough set (MGNRS). First, the concept of MGNRS on a single domain and dual domains was proposed. Then, their properties and operators were considered. We obtained that MGNRS on dual domains will degenerate into MGNRS on a single domain when the two domains are the same. Finally, a kind of special multi-criteria group decision making (MCGDM) problem was solved based on MGNRS on dual domains, and an example was given to show its feasibility.
\end{abstract}

Keywords: neutrosophic rough set; MGNRS; dual domains; inclusion relation; decision-making

\section{Introduction}

As we all know, Pawlak first proposed a rough set in 1982, which was a useful tool of granular computing. The relation is an equivalent in Pawlak's rough set. After that, many researchers proposed other types of rough set theory (see the work by the following authors [1-8]).

In 1965, Zadeh presented a new concept of the fuzzy set. After that, a lot of scholars studied it and made extensions. For example, Atanassov introduced an intuitionistic fuzzy set, which gives two degrees of membership of an element; it is a generalization of the fuzzy set. Smarandache introduced a neutrosophic set in 1998 [9,10], which was an extension of the intuitionistic fuzzy set. It gives three degrees of membership of an element (T.I.F). Smarandache and Wang [11] proposed the definition of a single valued neutrosophic set and studied its operators. Ye [12] proposed the definition of simplified neutrosophic sets and studied their operators. Zhang et al. [13] introduced a new inclusion relation of the neutrosophic set and told us when it was used by an example, and its lattice structure was studied. Garg and Nancy proposed neutrosophic operators and applied them to decision-making problems [14-16]. Now, some researchers have combined the fuzzy set and rough set and have achieved many running results, such as the fuzzy rough set [17] and rough fuzzy set. Broumi and Smarandache [18] proposed the definition of a rough neutrosophic set and studied their operators and properties. In 2016, Yang et al. [19] proposed the definition of single valued neutrosophic rough sets and studied their operators and properties.

Under the perspective of granular computing [20], the concept of a rough set is shown by the upper and lower approximations of granularity. In other words, the concept is represented by the 
known knowledge, which is defined by a single relationship. In fact, to meet the user's needs or achieve the goal of solving the problem, it is sometimes necessary to use multiple relational representation concepts on the domain, such as illustrated by the authors of [21]. In a grain calculation, an equivalence relation in the domain is a granularity, and a partition is considered as a granularity space [22]. The approximation that is defined by multiple equivalence relationships is a multi-granularity approximation and multiple partitions are considered as multi-granularity spaces; the resulting rough set is named a multi-granularity rough set, which has been proposed by Qian and Liang [23]. Recently, many scholars [24,25] have studied it and made extensions. Huang et al. [26] proposed the notion of intuitionistic fuzzy multi-granulation rough sets and studied their operators. Zhang et al. [27] introduced two new multi-granulation rough set models and investigated their operators. Yao et al. [28] made a summary about the rough set models on the multi-granulation spaces.

Although there have been many studies regarding multi-granulation rough set theory, there have been fewer studies about the multi-granulation rough set model on dual domains. Moreover, a multi-granulation rough set on dual domains is more convenient, for example, medical diagnosis in clinics [22,29]. The symptoms are the uncertainty index sets and the diseases are the decision sets. They are associated with each other, but they belong to two different domains. Therefore, it is necessary to use two different domains when solving the MCGDM problems. Sun et al. [29] discussed the multi-granulation rough set models based on dual domains; their properties were also obtained.

Although neutrosophic sets and multi-granulation rough sets are both useful tools to solve uncertainty problems, there are few research regarding their combination. In this paper, we proposed the definition of MGNRS as a rough set generated by multi-neutrosophic relations. It is useful to solve a kind of special group decision-making problem. We studied their properties and operations and then built a way to solve MCGDM problems based on the MGNRS theory on dual domains.

The structure of the article is as follows. In Section 2, some basic notions and operations are introduced. In Section 3, the notion of MGNRS is proposed and their properties are studied. In Section 4, the model of MGNRS on dual domains is proposed and their properties are obtained. Also, we obtained that MGNRS on dual domains will degenerate into MGNRS on a single domain when the two domains are same. In Section 5, an application of the MGNRS to solve a MCGDM problem was proposed. Finally, Section 6 concludes this paper and provides an outlook.

\section{Preliminary}

In this section, we review several basic concepts and operations of the neutrosophic set and multi-granulation rough set.

Definition 1 ([11]). A single valued neutrosophic set B is denoted by $\forall y \in Y$, as follows:

$$
B(y)=\left(T_{B}(y), I_{B}(y), F_{B}(y)\right)
$$

$T_{B}(y), I_{B}(y), F_{B}(y) \in[0,1]$ and satisfies $0 \leq T_{B}(y)+I_{B}(y)+F_{B}(y) \leq 3$.

As a matter of convenience, 'single valued neutrosophic set' is abbreviated to 'neutrosophic set' later. In this paper, $N S(Y)$ denotes the set of all single valued neutrosophic sets in $Y$, and $N R(Y \times Z)$ denotes the set of all of the neutrosophic relations in $Y \times Z$.

Definition 2 ([11]). If $A$ and $C$ are two neutrosophic sets, then the inclusion relation, union, intersection, and complement operations are defined as follows:

(1) $A \subseteq C$ iff $\forall y \in Y, T A(y) \leq T C(y), I A(y) \geq I C(y)$ and $F A(y) \geq F C(y)$

(2) $A^{c}=\left\{\left(y, F_{A}(y), 1-I_{A}(y), T_{A}(y)\right) \mid y \in Y\right\}$

(3) $A \cap C=\left\{\left(y, T_{A}(y) \wedge T_{C}(y), I_{A}(y) \vee I_{C}(y), F_{A}(y) \vee F_{C}(y)\right) \mid y \in Y\right\}$

(4) $A \cup C=\left\{\left(y, T_{A}(y) \vee T_{C}(y), I_{A}(y) \wedge I_{C}(y), F_{A}(y) \wedge F_{C}(y)\right) \mid y \in Y\right\}$ 
Definition 3 ([19]). If $(U, R)$ is a single valued neutrosophic approximation space. Then $\forall B \in S V N S(U)$, the lower approximation $\underline{N}(B)$ and upper approximation $\bar{N}(B)$ of B are defined as follows:

$$
\begin{gathered}
T_{\underline{N}(B)}(y)=\min _{z \in U}\left[\max \left(F_{R}(y, z), T_{B}(z)\right)\right], I_{\underline{N}(B)}(y)=\max _{z \in U}\left[\min \left(\left(1-I_{R}(y, z)\right), I_{B}(z)\right)\right], \\
F_{\underline{N}(B)}(y)=\max _{z \in U}\left[\min \left(T_{R}(y, z), F_{B}(z)\right)\right] \\
T_{\bar{N}(B)}(y)=\max _{z \in U}\left[\min \left(T_{R}(y, z), T_{B}(z)\right)\right], I_{\bar{N}(B)}(y)=\min _{z \in U}\left[\max \left(I_{R}(y, z), I_{B}(z)\right)\right], \\
F_{\bar{N}(B)}(y)=\min _{z \in U}\left[\max \left(F_{R}(y, z), F_{B}(z)\right)\right]
\end{gathered}
$$
to $(\mathrm{U}, \mathrm{R})$.

The pair $(\underline{N}(B), \bar{N}(B))$ is called the single valued neutrosophic rough set of $\mathrm{B}$, with respect

According to the operation of neutrosophic number in [16], the sum of two neutrosophic sets in $U$ is defined as follows.

Definition 4. If $C$ and $D$ are two neutrosophic sets in $U$, then the sum of $C$ and $D$ is defined as follows:

$$
C+D=\{<y, C(y) \oplus D(y)>I y \in U\}
$$

Definition 5 ([30]). If $b=\left(T_{b}, I_{b}, F_{b}\right)$ is a neutrosophic number, $n^{*}=\left(T_{b^{*}}, I_{b^{*}}, F_{b^{*}}\right)=(1,0,0)$ is an ideal neutrosophic number. Then, the cosine similarity measure is defined as follows:

$$
S\left(b, b^{*}\right)=\frac{T_{b} \cdot T_{b^{*}}+I_{b} \cdot I_{b^{*}}+F_{b} \cdot F_{b^{*}}}{\sqrt{T_{b}^{2}+I_{b}^{2}+F_{b}^{2}} \cdot \sqrt{\left(T_{b^{*}}\right)^{2}+\left(I_{b^{*}}\right)^{2}+\left(F_{b^{*}}\right)^{2}}}
$$

\section{Multi-Granulation Neutrosophic Rough Sets}

In this part, we propose the concept of MGNRS and study their characterizations. MGNRS is a rough set generated by multi-neutrosophic relations, and when all neutrosophic relations are same, MGNRS will degenerated to neutrosophic rough set.

Definition 6. Assume $U$ is a non-empty finite domain, and $R_{i}(1 \leq i \leq n)$ is the binary neutrosophic relation on $U$. Then, $\left(U, R_{i}\right)$ is called the multi-granulation neutrosophic approximation space (MGNAS).

Next, we present the multi-granulation rough approximation of a neutrosophic concept in an approximation space.

Definition 7. Let the tuple ordered set $\left(U, R_{i}\right)(1 \leq i \leq n)$ be a MGNAS. For any $B \in N S(U)$, the three memberships of the optimistic lower approximation $\underline{M}^{o}(B)$ and optimistic upper approximation $\bar{M}^{o}(B)$ in $\left(U, R_{i}\right)$ are defined, respectively, as follows:

$$
\begin{gathered}
T_{\underline{M}^{o}(B)}(y)=\max _{i=1}^{n} \min _{z \in U}\left(\max \left(F_{R_{i}}(y, z), T_{B}(z)\right)\right) I_{\underline{M}^{o}(B)}(y)=\min _{i=1}^{n} \max _{z \in U}\left(\min \left(\left(1-I_{R_{i}}(y, z)\right), I_{B}(z)\right)\right), \\
F_{\underline{M}^{o}(B)}(y)=\min _{i=1} \max _{z \in U}\left(\min \left(T_{R_{i}}(y, z), F_{B}(z)\right)\right), T_{\bar{M}^{o}(B)}(y)=\min _{i=1} \max _{z \in U}\left(\min \left(T_{R_{i}}(y, z), T_{B}(z)\right)\right), \\
I_{\bar{M}^{o}(B)}(y)=\max _{i=1} \min _{z \in U}\left(\max \left(I_{R_{i}}(y, z), I_{B}(z)\right)\right), F_{\bar{M}^{o}(B)}(y)=\max _{i=1} \min _{z \in U}\left(\max \left(F_{R_{i}}(y, z), F_{B}(z)\right)\right) .
\end{gathered}
$$

Then, $\underline{M}^{o}(B), \bar{M}^{o}(B) \in \mathrm{NS}(\mathrm{U})$. In addition, B is called a definable neutrosophic set on $\left(\mathrm{U}, \mathrm{R}_{\mathrm{i}}\right)$ when $\underline{M}^{o}(B)=\bar{M}^{o}(B)$. Otherwise, the pair $\left(\underline{M}^{o}(B), \bar{M}^{o}(B)\right)$ is called an optimistic MGNRS. 
Definition 8. Let the tuple ordered set $\left(U, R_{i}\right)(1 \leq i \leq n)$ be a MGNAS. For any $B \in N S(U)$, the three memberships of pessimistic lower approximation $\underline{M}^{p}(B)$ and pessimistic upper approximation $\bar{M}^{p}(B)$ in $\left(U, R_{i}\right)$ are defined, respectively, as follows:

$$
\begin{gathered}
T_{\underline{M}^{p}(B)}(y)=\min _{i=1}^{n} \min _{z \in U}\left(\max \left(F_{R_{i}}(y, z), T_{B}(z)\right)\right), I_{\underline{M}^{p}(B)}(y)=\max _{i=1}^{n} \max _{z \in U}\left(\min \left(\left(1-I_{R_{i}}(y, z)\right), I_{B}(z)\right)\right), \\
\underline{F}_{\underline{M}^{p}(B)}(y)=\max _{i=1} \max _{z \in U}\left(\min \left(T_{R_{i}}(y, z), F_{B}(z)\right)\right), T_{\bar{M}^{p}(B)}(y)=\max _{i=1} \max _{z \in U}\left(\min \left(T_{R_{i}}(y, z), T_{B}(z)\right)\right) \\
I_{\bar{M}^{p}(B)}(y)=\min _{i=1} \min _{z \in U}\left(\max \left(I_{R_{i}}(y, z), I_{B}(z)\right)\right), F_{\bar{M}^{p}(B)}(y)=\min _{i=1} \min _{z \in U}\left(\max \left(F_{R_{i}}(y, z), F_{B}(z)\right)\right) .
\end{gathered}
$$

Similarly, $B$ is called a definable neutrosophic set on $\left(U, R_{i}\right)$ when $\underline{M}^{p}(B)=\bar{M}^{p}(B)$. Otherwise, the pair $\left(\underline{M}^{p}(B), \bar{M}^{p}(B)\right)$ is called a pessimistic MGNRS.

Example 1. Define MGNAS $\left(U, R_{i}\right)$, where $U=\left\{z_{1}, z_{2}, z_{3}\right\}$ and $R_{i}(1 \leq i \leq 3)$ are given in Tables $1-3$

Table 1. Neutrosophic relation $R_{1}$.

\begin{tabular}{llll}
\hline $\boldsymbol{R}_{\mathbf{1}}$ & $z_{\mathbf{1}}$ & $z_{2}$ & $z_{3}$ \\
\hline$z_{1}$ & $(0.4,0.5,0.4)$ & $(0.5,0.7,0.1)$ & $(1,0.8,0.8)$ \\
$z_{2}$ & $(0.5,0.6,1)$ & $(0.2,0.6,0.4)$ & $(0.9,0.2,0.4)$ \\
$z_{3}$ & $(1,0.2,0)$ & $(0.8,0.9,1)$ & $(0.6,1,0)$ \\
\hline
\end{tabular}

Table 2. Neutrosophic relation $R_{2}$.

\begin{tabular}{llll}
\hline $\boldsymbol{R}_{\mathbf{2}}$ & $z_{\mathbf{1}}$ & $z_{2}$ & $z_{3}$ \\
\hline$z_{1}$ & $(0.9,0.2,0.4)$ & $(0.3,0.9,0.1)$ & $(0.1,0.7,0)$ \\
$z_{2}$ & $(0.4,0.5,0.1)$ & $(0,0.1,0.7)$ & $(1,0.8,0.8)$ \\
$z_{3}$ & $(1,0.5,0)$ & $(0.4,0.4,0.2)$ & $(0.1,0.5,0.4)$ \\
\hline
\end{tabular}

Table 3. Neutrosophic relation $R_{3}$.

\begin{tabular}{llll}
\hline $\boldsymbol{R}_{\mathbf{3}}$ & $z_{\mathbf{1}}$ & $z_{\mathbf{2}}$ & $z_{3}$ \\
\hline$z_{1}$ & $(0.7,0.7,0)$ & $(0.4,0.8,0.9)$ & $(1,0.4,0.5)$ \\
$z_{2}$ & $(0.8,0.2,0.1)$ & $(1,0.1,0.8)$ & $(0.1,0.3,0.5)$ \\
$z_{3}$ & $(0,0.8,1)$ & $(1,0,1)$ & $(1,1,0)$ \\
\hline
\end{tabular}

Suppose a neutrosophic set on $U$ is as follows: $C\left(z_{1}\right)=(0.2,0.6,0.4), C\left(z_{2}\right)=(0.5,0.4,1)$, $C\left(z_{3}\right)=(0.7,0.1,0.5)$; by Definitions 7 and 8 , we can get the following:

$$
\begin{aligned}
& \underline{M}^{o}(C)\left(z_{1}\right)=(0.4,0.3,0.4), \underline{M}^{o}(C)\left(z_{2}\right)=(0.5,0.4,0.5), \underline{M}^{o}(C)\left(z_{3}\right)=(0.7,0.4,0.4) \\
& \bar{M}^{o}(C)\left(z_{1}\right)=(0.3,0.6,0.4), \bar{M}^{o}(C)\left(z_{2}\right)=(0.5,0.4,0.5), \bar{M}^{o}(C)\left(z_{3}\right)=(0.4,0.6,0.5) \\
& \underline{M}^{p}(C)\left(z_{1}\right)=(0.2,0.6,0.5), \underline{M}^{p}(C)\left(z_{2}\right)=(0.2,0.6,0.1), \underline{M}^{p}(C)\left(z_{3}\right)=(0.2,0.6,0.1) \\
& \left.\bar{M}^{p}(C)\left(z_{1}\right)=(0.7,0.4,0.4), \bar{M}^{p}(C)\left(z_{2}\right)=(0.7,0.2,0.4), z_{3}\right)=(0.7,0.4,0.4)
\end{aligned}
$$

Proposition 1. Assume $\left(U, R_{i}\right)$ is $M G N A S, R_{i}(1 \leq i \leq n)$ is the neutrosophic relations. $\forall C \in N S(U), \underline{M}^{o}(C)$ and $\bar{M}^{O}(C)$ are the optimistic lower and upper approximation of $C$. Then,

$$
\underline{M}^{o}(C)=\bigcup_{i=1}^{n} \underline{N}(C) \bar{M}^{o}(C)=\bigcap_{i=1}^{n} \bar{N}(C)
$$

where

$$
\underline{N}(C)(y)=\bigcap_{z \in U}\left(R_{i}^{c}(y, z) \cup C(z)\right), \bar{N}(C)(y)=\cup_{z \in U}\left(R_{i}(y, z) \cap C(z)\right)
$$

Proof. They can be proved by Definitions 7. 
Proposition 2. Assume $\left(U, R_{i}\right)$ be MGNAS, $R_{i}(1 \leq i \leq n)$ be neutrosophic relations. $\forall C \in N S(U), \underline{M}^{p}(C)$ and $\bar{M}^{p}(C)$ are the pessimistic lower and upper approximation of $C$. Then

$$
\underline{M}^{p}(C)=\stackrel{n}{n}=1_{n}^{n}(C) \bar{M}^{p}(C)=\cup_{i=1}^{n} \bar{N}(C)
$$

where

$$
\underline{N}(C)(y)=\underset{z \in U}{\cap}\left(R_{i}^{c}(y, z) \cup C(z)\right), \bar{N}(C)(y)=\cup_{z \in U}\left(R_{i}(y, z) \cap C(z)\right)
$$

Proof. Proposition 2 can be proven by Definition 8 .

Proposition 3. Assume $\left(U, R_{i}\right)$ is MGNAS, $R_{i}(1 \leq i \leq n)$ is the neutrosophic relations. $\forall C, D \in N S(U)$, we have the following:

(1) $\underline{M}^{o}(C)=\sim \bar{M}^{o}(\sim C), \underline{M}^{p}(C)=\sim \bar{M}^{p}(\sim C) ;$

(2) $\bar{M}^{o}(C)=\sim \underline{M}^{o}(\sim C), \bar{M}^{p}(C)=\sim \underline{M}^{p}(\sim C)$;

(3) $\quad \underline{M}^{o}(C \cap D)=\underline{M}^{o}(C) \cap \underline{M}^{o}(D), \underline{M}^{p}(C \cap D)=\underline{M}^{p}(C) \cap \underline{M}^{p}(D)$;

(4) $\bar{M}^{o}(C \cup D)=\bar{M}^{o}(C) \cup \bar{M}^{o}(D), \bar{M}^{p}(C \cup D)=\bar{M}^{p}(C) \cup \bar{M}^{p}(D)$;

(5) $C \subseteq D \Rightarrow \underline{M}^{o}(C) \subseteq \underline{M}^{o}(D), \underline{M}^{p}(C) \subseteq \underline{M}^{p}(D)$;

(6) $C \subseteq D \Rightarrow \bar{M}^{o}(C) \subseteq \bar{M}^{o}(D), \bar{M}^{p}(C) \subseteq \bar{M}^{p}(D)$;

(7) $\quad \underline{M}^{o}(C \cup D) \supseteq \underline{M}^{o}(C) \cup \underline{M}^{o}(D), \underline{M}^{p}(C \cup D) \supseteq \underline{M}^{p}(C) \cup \underline{M}^{p}(D)$;

(8) $\overline{\bar{M}}^{o}(C \cap D) \subseteq \bar{M}^{o}(C) \cap \bar{M}^{o}(D), \bar{M}^{p}(C \cap D) \subseteq \bar{M}^{p}(C) \cap \bar{M}^{p}(D)$.

Proof. (1), (2), (5), and (6) can be taken directly from Definitions 7 and 8. We only show (3), (4), (7), and (8).

(3) From Proposition 1, we have the following:

$$
\begin{aligned}
\underline{M}^{o}(C \cap D)(y)= & \bigcup_{i=1}^{n}\left(\bigcap_{z \in U}\left(R_{i}^{c}(y, z) \cup(C \cap D)(z)\right)\right) \\
& =\bigcup_{i=1}^{n}\left(\bigcap_{z \in U}\left(\left(R_{i}^{c}(y, z) \cup C(z)\right) \cap\left(R_{i}^{c}(y, z) \cup D(z)\right)\right)\right) \\
& =\left(\bigcup_{i=1}^{n}\left(\bigcap_{z \in U}\left(R_{i}^{c}(y, z) \cup C(z)\right)\right)\right) \cap\left(\bigcup_{i=1}^{n}\left(\bigcap_{z \in U}\left(R_{i}^{c}(y, z) \cup D(z)\right)\right)\right) \\
& =\underline{M}^{o} C(y) \cap \underline{M}^{o} D(y) .
\end{aligned}
$$

Similarly, from Proposition 2, we can get the following:

$$
\underline{M}^{p}(C \cap D)(y)=\underline{M}^{p} C(y) \cap \underline{M}^{p} D(y) .
$$

(4) According to Propositions 1 and 2, in the same way as (3), we can get the proof.

(7) From Definition 7, we have the following:

$$
\begin{aligned}
T_{\underline{M}^{o}(C \cup D)}(y) & =\max _{i=1}^{n} \min _{z \in U}\left\{\max \left[F_{R_{i}}(y, z),\left(\max \left(T_{C}(z), T_{D}(z)\right)\right)\right]\right\} \\
& =\max _{i=1} \min _{z \in U}\left\{\max \left[\left(\max \left(F_{R_{i}}(y, z), T_{C}(z)\right)\right),\left(\max \left(F_{R_{i}}(y, z), T_{D}(z)\right)\right)\right]\right\} \\
& \geq \max \left\{\left[\max _{i=1}^{n} \min _{z \in U}\left(\max \left(F_{R_{i}}(y, z), T_{C}(z)\right)\right)\right],\left[\max _{i=1}^{n} \min _{z \in U}\left(\max \left(F_{R_{i}}(y, z), T_{D}(z)\right)\right)\right]\right\} \\
& =\max \left(T_{\underline{M}^{o}(C)}(y), T_{\underline{M}^{o}(D)}(y)\right) .
\end{aligned}
$$




$$
\begin{aligned}
& I_{\underline{M}^{o}(C \cup D)}(y)=\min _{i=1}^{n} \max _{z \in U}\left\{\min \left[\left(1-I_{R_{i}}(y, z)\right),\left(\min \left(I_{C}(z), I_{D}(z)\right)\right)\right]\right\} \\
& =\min _{i=1}^{n} \max _{z \in U}\left\{\min \left[\left(\min \left(\left(1-I_{R_{i}}(y, z)\right), I_{C}(z)\right)\right),\left(\min \left(\left(1-I_{R_{i}}(y, z)\right), I_{D}(z)\right)\right)\right]\right\} \\
& \leq \min \left\{\left[\min _{i=1}^{n} \max _{z \in U}\left(\min \left(\left(1-I_{R_{i}}(y, z)\right), I_{C}(z)\right)\right)\right],\left[\min _{i=1}^{n} \max _{z \in U}\left(\min \left(\left(1-I_{R_{i}}(y, z)\right), I_{D}(z)\right)\right)\right]\right\} \\
& =\min \left(\underline{I}_{\underline{M}^{o}(C)}(y), I_{\underline{M}^{o}(D)}(y)\right) \text {. } \\
& F_{\underline{M}^{o}(C \cup D)}(y)=\min _{\substack{i=1 \\
n}}^{n} \max _{z \in U}\left\{\min \left[T_{R_{i}}(y, z),\left(\min \left(F_{C}(z), F_{D}(z)\right)\right)\right]\right\} \\
& =\min _{i=1}^{n} \max _{z \in U}\left\{\min \left[\min \left(T_{R_{i}}(y, z), F_{C}(z)\right)\right],\left[\min \left(T_{R_{i}}(y, z), F_{D}(z)\right)\right]\right\} \\
& \leq \min \left\{\left[\min _{i=1}^{n} \max _{z \in U}\left(\min \left(T_{R_{i}}(y, z), F_{C}(z)\right)\right)\right],\left[\min _{i=1}^{n} \max _{z \in U}\left(\min \left(T_{R_{i}}(y, z), F_{D}(z)\right)\right)\right]\right\} \\
& =\min \left(\underline{F}_{\underline{M}^{o}(C)}(y), \underline{F}_{\underline{M}^{o}(D)}(y)\right) \text {. }
\end{aligned}
$$

Hence, $\underline{M}^{o}(C \cup D) \supseteq \underline{M}^{o}(C) \cup \underline{M}^{o}(D)$.

Also, according to Definition 8, we can get $\underline{M}^{p}(C \cup D) \supseteq \underline{M}^{p}(C) \cup \underline{M}^{p}(D)$.

(8) From Definition 7, we have the following:

$$
\begin{aligned}
& T_{\bar{M}^{o}(C \cap D)}(y)=\min _{i=1}^{n} \max _{z \in U}\left\{\min \left[T_{R_{i}}(y, z),\left(\min \left(T_{C}(z), T_{D}(z)\right)\right)\right]\right\} \\
& =\min _{i=1} \max _{z \in U}\left\{\min \left[\left(\min \left(T_{R_{i}}(y, z), T_{C}(z)\right)\right),\left(\min \left(T_{R_{i}}(y, z), T_{D}(z)\right)\right)\right]\right\} \\
& \leq \min \left\{\left[\min _{i=1}^{n} \max _{z \in U}\left(\min \left(T_{R_{i}}(y, z), T_{C}(z)\right)\right)\right],\left[\min _{i=1}^{n} \max _{z \in U}\left(\min \left(T_{R_{i}}(y, z), T_{D}(z)\right)\right)\right]\right\} \\
& =\min \left(T_{\bar{M}^{o}(C)}(y), T_{\bar{M}^{o}(D)}(y)\right) \text {. } \\
& I_{\bar{M}^{o}(C \cap D)}(y)=\max _{i=1}^{n} \min _{z \in U}\left\{\max \left[I_{R_{i}}(y, z),\left(\max \left(I_{C}(z), I_{D}(z)\right)\right)\right]\right\} \\
& =\max _{i=1}^{n} \min _{z \in U}\left\{\max \left[\left(\max \left(I_{R_{i}}(y, z), I_{C}(z)\right)\right),\left(\max \left(I_{R_{i}}(y, z), I_{D}(z)\right)\right)\right]\right\} \\
& \leq \min \left\{\left[\max _{i=1}^{n} \min _{z \in U}\left(\max \left(I_{R_{i}}(y, z), I_{C}(z)\right)\right)\right],\left[\max _{i=1}^{n} \min _{z \in U}\left(\max \left(I_{R_{i}}(y, z), I_{D}(z)\right)\right)\right]\right\} \\
& =\min \left(I_{\bar{M}^{o}(C)}(y), I_{\bar{M}^{o}(D)}(y)\right) \text {. } \\
& F_{\bar{M}^{o}(C \cap D)}(y)=\max _{i=1}^{n} \min _{z \in U}\left[F_{R_{i}}(y, z) \vee\left(F_{C}(z) \vee F_{D}(z)\right)\right] \\
& =\max _{i=1}^{n} \min _{z \in U}\left[\left(F_{R_{i}}(y, z) \vee F_{C}(z)\right) \vee\left(F_{R_{i}}(y, z) \vee F_{D}(z)\right)\right] \\
& \geq\left[\max _{i=1}^{n} \min _{z \in U}\left(F_{R_{i}}(y, z) \vee F_{C}(z)\right)\right] \vee\left[\max _{i=1}^{n} \min _{z \in U}\left(F_{R_{i}}(y, z) \vee F_{D}(z)\right)\right] \\
& =\max \left(F_{\bar{M}^{0}(C)}(y), F_{\bar{M}^{o}(D)}(y)\right) \text {. }
\end{aligned}
$$

Hence, $\bar{M}^{o}(C \cap D) \subseteq \bar{M}^{o}(C) \cap \bar{M}^{o}(D)$.

Similarly, according Definition 8 , we can get $\bar{M}^{p}(C \cap D) \subseteq \bar{M}^{p}(C) \cap \bar{M}^{p}(D)$.

Next, we will give an example to show that maybe $\underline{M}^{o}(C \cup D) \neq \underline{M}^{o}(C) \cup \underline{M}^{o}(D)$.

Example 2. Define MGNAS $\left(U, R_{i}\right)$, where $U=\left\{z_{1}, z_{2}, z_{3}\right\}$ and $R_{i}(1 \leq i \leq 3)$ are given in Example 1 .

Suppose there are two neutrosophic sets on universe $U$, as follows: $C\left(z_{1}\right)=(0.5,0.1,0.2), C\left(z_{2}\right)=(0.5$, $0.3,0.2), C\left(z_{3}\right)=(0.6,0.2,0.1), D\left(z_{1}\right)=(0.7,0.2,0.1), D\left(z_{2}\right)=(0.4,0.2,0.1), D\left(z_{3}\right)=(0.2,0.2,0.5)$, we have $(C \cup D)\left(z_{1}\right)=(0.7,0.1,0.1),(C \cup D)\left(z_{2}\right)=(0.5,0.2,0.1),(C \cup D)\left(z_{3}\right)=(0.6,0.2,0.1),(C \cap D)\left(z_{1}\right)=(0.5,0.1$, 
$0.2),(C \cap D)\left(z_{2}\right)=(0.4,0.2,0.2),(C \cap D)\left(z_{3}\right)=(0.2,0.2,0.5)$. Then, from Definitions 7 and 8 , we can get the following:

$$
\begin{gathered}
\underline{M}^{o}(C)\left(z_{1}\right)=(0.5,0,0.2), \underline{M}^{o}(C)\left(z_{2}\right)=(0.5,0.1,0.2), \underline{M}^{o}(C)\left(z_{3}\right)=(0.5,0.1,0.2) ; \\
\underline{M}^{o}(D)\left(z_{1}\right)=(0.4,0,0.1), \underline{M}^{o}(D)\left(z_{2}\right)=(0.2,0.1,0.2), \underline{M}^{o}(D)\left(z_{3}\right)=(0.4,0.1,0.2) ; \\
\underline{M}^{o}(C \cup D)\left(z_{1}\right)=(0.5,0,0.1), \underline{M}^{o}(C \cup D)\left(z_{2}\right)=(0.5,0.1,0.1), \underline{M}^{o}(C \cup D)\left(z_{3}\right)=(0.5,0.1,0.1) \\
\left(\underline{M}^{o}(C) \cup \underline{M}^{o}(D)\right)\left(z_{1}\right)=(0.5,0,0.1),\left(\underline{M}^{o}(C) \cup \underline{M}^{o}(D)\right)\left(z_{2}\right)=(0.5,0.1,0.2), \\
\left(\underline{M}^{o}(C) \cup \underline{M}^{o}(D)\right)\left(z_{3}\right)=(0.5,0.1,0.2)
\end{gathered}
$$

So, $\underline{M}^{o}(C \cup D) \neq \underline{M}^{o}(C) \cup \underline{M}^{o}(D)$.

Also, there are examples to show that maybe $\underline{M}^{p}(C \cup D) \neq \underline{M}^{p}(C) \cup \underline{M}^{p}(D)$,

$\bar{M}^{o}(C \cap D) \neq \bar{M}^{o}(C) \cap \bar{M}^{o}(D), \bar{M}^{p}(C \cap \bar{D}) \neq \bar{M}^{p}(C) \cap \bar{M}^{p}(D)$. We do not say anymore here.

\section{Multi-Granulation Neutrosophic Rough Sets on Dual Domains}

In this section, we propose the concept of MGNRS on dual domains and study their characterizations. Also, we obtain that the MGNRS on dual domains will degenerate into MGNRS, defined in Section 3, when the two domains are same.

Definition 9. Assume that $U$ and $V$ are two domains, and $R_{i} \in N S(U \times V)(1 \leq i \leq n)$ is the binary neutrosophic relations. The triple ordered set $\left(U, V, R_{i}\right)$ is called the (two-domain) MGNAS.

Next, we present the multi-granulation rough approximation of a neutrosophic concept in an approximation space on dual domains.

Definition 10. Let $\left(U, V, R_{i}\right)(1 \leq i \leq n)$ be (two-domain) MGNAS. $\forall B \in N S(V)$ and $y \in U$, the three memberships of the optimistic lower and upper approximation $\underline{M}^{o}(B), \bar{M}^{o}(B)$ in $\left(U, V, R_{i}\right)$ are defined, respectively, as follows:

$$
\begin{gathered}
T_{\underline{M}^{o}(B)}(y)=\max _{i=1}^{n} \min _{z \in V}\left[\max \left(F_{R_{i}}(y, z), T_{B}(z)\right)\right] I_{\underline{M}^{o}(B)}(y)=\min _{i=1}^{n} \max _{z \in V}\left[\min \left(\left(1-I_{R_{i}}(y, z)\right), I_{B}(z)\right)\right] \\
F_{\underline{M}^{o}(B)}(y)=\min _{i=1}^{n} \max _{z \in V}\left[\min \left(T_{R_{i}}(y, z), F_{B}(z)\right)\right] T_{\bar{M}^{o}(B)}(y)=\min _{i=1}^{n} \max _{z \in V}\left[\min \left(T_{R_{i}}(y, z), T_{B}(z)\right)\right] \\
I_{\sum_{i=1}^{n} R_{i}}^{o}(B) \\
\ln _{i=1} \min _{z \in V}\left[\max \left(I_{R_{i}}(y, z), I_{B}(z)\right)\right] F_{\bar{M}^{o}(B)}(y)=\max _{i=1} \min _{z \in V}\left[\max \left(F_{R_{i}}(y, z), F_{B}(z)\right)\right]
\end{gathered}
$$

Then $\underline{M}^{o}(B), \bar{M}^{o}(B) \in N S(U)$. In addition, $B$ is called a definable neutrosophic set on $\left(U, V, R_{i}\right)$ on dual domains when $\underline{M}^{o}(B)=\bar{M}^{o}(B)$. Otherwise, the pair $\left(\underline{M}^{o}(B), \bar{M}^{o}(B)\right)$ is called an optimistic MGNRS on dual domains.

Definition 11. Assume $\left(U, V, R_{i}\right)(1 \leq i \leq n)$ is (two-domain) MGNAS. $\forall B \in N S(V)$ and $y \in U$, the three memberships of the pessimistic lower and upper approximation $\underline{M}^{p}(B), \bar{M}^{p}(B)$ in $\left(U, V, R_{i}\right)$ are defined, respectively, as follows:

$$
\begin{gathered}
\underline{T}_{\underline{M}^{p}(B)}(y)=\min _{i=1}^{n} \min _{z \in V}\left[\max \left(F_{R_{i}}(y, z), T_{B}(z)\right)\right], \underline{I}_{\underline{M}^{p}(B)}(y)=\max _{i=1}^{n} \max _{z \in V}\left[\min \left(\left(1-I_{R_{i}}(y, z)\right), I_{B}(z)\right)\right], \\
F_{\underline{M}^{p}(B)}(y)=\max _{i=1}^{n} \max _{z \in V}\left[\min \left(T_{R_{i}}(y, z), F_{B}(z)\right)\right], T_{\bar{M}^{p}(B)}(y)=\max _{i=1}^{n} \max _{z \in V}\left[\min \left(T_{R_{i}}(y, z), T_{B}(z)\right)\right], \\
I_{\bar{M}^{p}(B)}(y)=\min _{i=1} \min _{z \in V}\left[\max \left(I_{R_{i}}(y, z), I_{B}(z)\right)\right], F_{\bar{M}^{p}(B)}(y)=\min _{i=1} \min _{z \in V}\left[\max \left(F_{R_{i}}(y, z), F_{B}(z)\right)\right] .
\end{gathered}
$$


Then, $B$ is called a definable neutrosophic set on $\left(U, V, R_{i}\right)$ when $\underline{M}^{p}(B)=\bar{M}^{p}(B)$. Otherwise, the pair $\left(\underline{M}^{p}(B), \bar{M}^{p}(B)\right)$ is called a pessimistic MGNRS on dual domains.

Remark 1. Note that if $U=V$, then the optimistic and pessimistic MGNRS on the dual domains will be the same with the optimistic and pessimistic MGNRS on a single domain, which is defined in Section 3

Proposition 4. Assume $\left(U, V, R_{i}\right)(1 \leq i \leq n)$ is (two-domain) MGNAS, $R_{i}(1 \leq i \leq n)$ is the neutrosophic relations. $\forall C, D \in N S(U)$, we have the following:

(1) $\underline{M}^{o}(C)=\sim \bar{M}^{o}(\sim C), \underline{M}^{p}(C)=\sim \bar{M}^{p}(\sim C)$;

(2) $\bar{M}^{o}(C)=\sim \underline{M}^{o}(\sim C), \bar{M}^{p}(C)=\sim \underline{M}^{p}(\sim C)$;

(3) $\underline{M}^{o}(C \cap D)=\underline{M}^{o}(C) \cap \underline{M}^{o}(D), \underline{M}^{p}(C \cap D)=\underline{M}^{p}(C) \cap \underline{M}^{p}(D)$;

(4) $\bar{M}^{o}(C \cup D)=\bar{M}^{o}(C) \cup \bar{M}^{o}(D), \bar{M}^{p}(C \cup D)=\bar{M}^{p}(C) \cup \bar{M}^{p}(D)$;

(5) $C \subseteq D \Rightarrow \underline{M}^{o}(C) \subseteq \underline{M}^{o}(D), \underline{M}^{p}(C) \subseteq \underline{M}^{p}(D)$;

(6) $C \subseteq D \Rightarrow \bar{M}^{o}(C) \subseteq \bar{M}^{o}(D), \bar{M}^{p}(C) \subseteq \bar{M}^{p}(D)$;

(7) $\quad \underline{M}^{o}(C \cup D) \supseteq \underline{M}^{o}(C) \cup \underline{M}^{o}(D), \underline{M}^{p}(C \cup D) \supseteq \underline{M}^{p}(C) \cup \underline{M}^{p}(D)$;

(8) $\bar{M}^{o}(C \cap D) \subseteq \bar{M}^{o}(C) \cap \bar{M}^{o}(D), \bar{M}^{p}(C \cap D) \subseteq \bar{M}^{p}(C) \cap \bar{M}^{p}(D)$.

Proof. These propositions can be directly proven from Definitions 10 and 11.

\section{An Application of Multi-Granulation Neutrosophic Rough Set on Dual Domains}

Group decision making [31] is a useful way to solve uncertainty problems. It has developed rapidly since it was first proposed. Its essence is that in the decision-making process, multiple decision makers (experts) are required to participate and negotiate in order to settle the corresponding decision-making problems. However, with the complexity of the group decision-making problems, what we need to deal with is the multi-criteria problems, that is, multi-criteria group decision making (MCGDM). The MCGDM problem is to select or rank all of the feasible alternatives in multiple, interactive, and conflicting standards.

In this section, we build a neo-way to solve a kind of special MCGDM problem using the MGNRS theory. We generated the rough set according the multi-neutrosophic relations and then used it to solve the decision-making problems. We show the course and methodology of it.

\subsection{Problem Description}

Firstly, we describe the considered problem and we show it using a MCGDM example of houses selecting.

Let $U=\left\{x_{1}, x_{2}, \ldots, x_{m}\right\}$ be the decision set, where $x_{1}$ represents very good, $x_{2}$ represents good, $x_{3}$ represents less good, $\ldots$, and $x_{m}$ represents not good. Let $V=\left\{y_{1}, y_{2}, \ldots, y_{n}\right\}$ be the criteria set to describe the given house, where $y_{1}$ represents texture, $y_{2}$ represents geographic location, $y_{3}$ represents price, $\ldots$, and $y_{n}$ represents solidity. Suppose there are $k$ evaluation experts and all of the experts give their own evaluation for criteria set $y_{j}\left(y_{j} \in V\right)(j=1,2, \ldots, n)$, regarding the decision set elements $x_{i}\left(x_{i} \in U\right)(i=1,2, \ldots, m)$. In this paper, let the evaluation relation $R_{1}, R_{2}, \ldots, R_{k}$ between $V$ and $U$ given by the experts, be the neutrosophic relation, $R_{1}, R_{2}, \ldots, R_{k} \in S N S(U \times V)$. That is, $R_{l}\left(x_{i}, y_{j}\right)$ $(l=1,2, \ldots, k)$ represents the relation of the criteria set $y_{j}$ and the decision set element $x_{i}$, which is given by expert $l$, based on their own specialized knowledge and experience. For a given customer, the criterion of the customer is shown using a neutrosophic set, $C$, in $V$, according to an expert's opinion. Then, the result of this problem is to get the opinion of the given house for the customer.

Then, we show the method to solve the above problem according to the theory of optimistic and pessimistic MGNRS on dual domains. 


\subsection{New Method}

In the first step, we propose the multi-granulation neutrosophic decision information system based on dual domains for the above problem.

According to Section $5.1^{\prime}$ s description, we can get the evaluation of each expert as a neutrosophic relation. Then, all of the binary neutrosophic relations $R_{l}$ given by all of the experts construct a relation set $\mathcal{R}$ (i.e., $R_{l} \in \mathcal{R}$ ). Then, we get the multi-granulation neutrosophic decision information systems based on dual domains, denoted by $(U, V, \mathcal{R})$.

Secondly, we compute $\underline{M}^{o}(C), \bar{M}^{o}(C), \underline{M}^{p}(C), \bar{M}^{p}(C)$ for the given customer, regarding $(U, V, \mathcal{R})$.

Thirdly, according to Definition 4, we computed the sum of the optimistic and pessimistic multi-granulation neutrosophic lower and upper approximation.

Next, according Definition 5, we computed the cosine similarity measure. Define the choice $x *$ with the idea characteristics value $\alpha *=(1,0,0)$ as the ideal choice. The bigger the value of $S\left(\alpha_{x_{i}}, \alpha^{*}\right)$ is, the closer the choice $x_{i}$ with the ideal alternative $x *$, so the better choice $x_{i}$ is.

Finally, we compared $S\left(\alpha_{x_{i}}, \alpha^{*}\right)$ and ranked all of the choices that the given customer can choose from and we obtained the optimal choice.

\subsection{Algorithm and Pseudo-Code}

In this section, we provide the algorithm and pseudo-code given in table Algorithm 1.

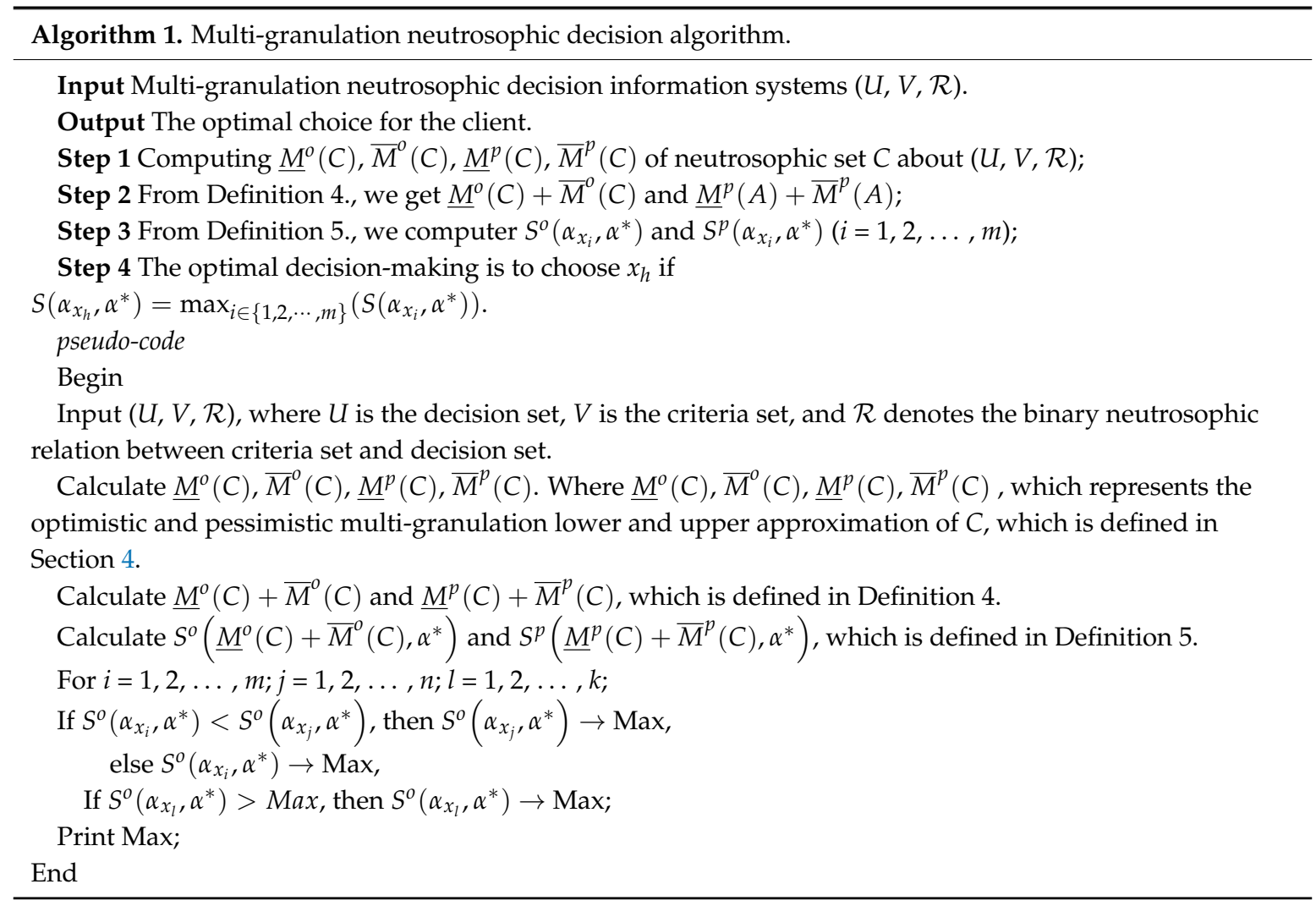

\subsection{An Example}

In this section, we used Section 5.2's way of solving a MCGDM problem, using the example of buying houses.

Let $V=\left\{y_{1}, y_{2}, y_{3}, y_{4}\right\}$ be the criteria set, where $y_{1}$ represents the texture, $y_{2}$ represents the geographic location, $y_{3}$ represents the price, and $y_{4}$ represents the solidity. Let $U=\left\{z_{1}, z_{2}, z_{3}, z_{4}\right\}$ be a decision set, where $z_{1}$ represents very good, $z_{2}$ represents good, $z_{3}$ represents less good, and $z_{4}$ represents not good. 
Assume that there are three experts. They provide their opinions about all of the criteria sets $y_{j}$ $\left(y_{j} \in V\right)(j=1,2,3,4)$ regarding the decision set elements $z_{i}\left(x_{i} \in U\right)(i=1,2,3,4)$. Like the discussion in Section 5.1, the experts give three evaluation relations, $R_{1}, R_{2}$, and $R_{3}$, which are neutrosophic relations between $V$ and $U$, that is, $R_{1}, R_{2}, R_{3} \in N R(U \times V)$. $T_{R k}\left(z_{i}, y_{j}\right)$ shows the expert, $k$, give the truth membership of $y_{j}$ to $z_{i} ; I_{R k}\left(z_{i}, y_{j}\right)$ shows the expert, $k$, give the indeterminacy membership of $y_{j}$ to $z_{i} ; F_{R k}\left(z_{i}, y_{j}\right)$ shows the expert, $k$, give the falsity membership of $y_{j}$ to $z_{i}$. For example, the first value $(0.2,0.3,0.4)$ in Table 4 , of 0.2 shows that the truth membership of the texture for the given house is very good, 0.3 shows that the indeterminacy membership of the texture for the given house is very good, and 0.4 shows that the falsity membership of the texture for the given house is very good.

Table 4. Neutrosophic relation $R_{1}$.

\begin{tabular}{lllll}
\hline $\boldsymbol{R}_{\mathbf{1}}$ & $\boldsymbol{y}_{\mathbf{1}}$ & $\boldsymbol{y}_{\mathbf{2}}$ & $\boldsymbol{y}_{\mathbf{3}}$ & $\boldsymbol{y}_{\mathbf{4}}$ \\
\hline$z_{1}$ & $(0.2,0.3,0.4)$ & $(0.3,0.5,0.4)$ & $(0.4,0.6,0.2)$ & $(0.1,0.3,0.5)$ \\
$z_{2}$ & $(0.8,0.7,0.1)$ & $(0.2,0.5,0.6)$ & $(0.6,0.6,0.7)$ & $(0.4,0.6,0.3)$ \\
$z_{3}$ & $(0.5,0.7,0.2)$ & $(0.6,0.2,0.1)$ & $(1,0.9,0.4)$ & $(0.5,0.4,0.3)$ \\
$z_{4}$ & $(0.4,0.6,0.3)$ & $(0.5,0.5,0.4)$ & $(0.3,0.8,0.4)$ & $(0.2,0.9,0.8)$ \\
\hline
\end{tabular}

So, we build the multi-granulation neutrosophic decision information system $(U, V, \mathcal{R})$ for the example.

Assume that the three experts give three evaluation relations, the results are given in Tables 4-6.

Table 5. Neutrosophic relation $R_{2}$.

\begin{tabular}{lllll}
\hline $\boldsymbol{R}_{\mathbf{2}}$ & $\boldsymbol{y}_{\mathbf{1}}$ & $\boldsymbol{y}_{\mathbf{2}}$ & $\boldsymbol{y}_{\mathbf{3}}$ & $\boldsymbol{y}_{\mathbf{4}}$ \\
\hline$z_{1}$ & $(0.3,0.4,0.5)$ & $(0.6,0.7,0.2)$ & $(0.1,0.8,0.3)$ & $(0.5,0.3,0.4)$ \\
$z_{2}$ & $(0.5,0.5,0.4)$ & $(1,0,1)$ & $(0.8,0.1,0.8)$ & $(0.7,0.8,0.5)$ \\
$z_{3}$ & $(0.7,0.2,0.1)$ & $(0.3,0.5,0.4)$ & $(0.6,0.1,0.4)$ & $(1,0,0)$ \\
$z_{4}$ & $(1,0.2,0)$ & $(0.8,0.1,0.5)$ & $(0.1,0.2,0.7)$ & $(0.2,0.2,0.8)$ \\
\hline
\end{tabular}

Table 6. Neutrosophic relation $R_{3}$.

\begin{tabular}{lllll}
\hline $\boldsymbol{R}_{\mathbf{3}}$ & $\boldsymbol{y}_{\mathbf{1}}$ & $\boldsymbol{y}_{\mathbf{2}}$ & $\boldsymbol{y}_{\mathbf{3}}$ & $\boldsymbol{y}_{\mathbf{4}}$ \\
\hline$z_{1}$ & $(0.6,0.2,0.2)$ & $(0.3,0.1,0.7)$ & $(0,0.2,0.9)$ & $(0.8,0.3,0.2)$ \\
$z_{2}$ & $(0.1,0.1,0.7)$ & $(0.2,0.3,0.8)$ & $(0.7,0.1,0.2)$ & $(0,0,1)$ \\
$z_{3}$ & $(0.8,0.4,0.1)$ & $(0.9,0.5,0.3)$ & $(0.2,0.1,0.6)$ & $(0.7,0.2,0.3)$ \\
$z_{4}$ & $(0.6,0.2,0.2)$ & $(0.2,0.2,0.8)$ & $(1,1,0)$ & $(0.5,0.3,0.1)$ \\
\hline
\end{tabular}

Assume $C$ is the customer's evaluation for each criterion in $V$, and is given by the following:

$$
C\left(y_{1}\right)=(0.6,0.5,0.5), C\left(y_{2}\right)=(0.7,0.3,0.2), C\left(y_{3}\right)=(0.4,0.5,0.9), C\left(y_{4}\right)=(0.3,0.2,0.6) .
$$

From Definitions 10 and 11, we can compute the following:

$$
\begin{gathered}
\underline{M}^{o}(C)\left(z_{1}\right)=(0.4,0.5,0.4), \frac{M^{o}(C)\left(z_{2}\right)=(0.5,0.4,0.6), \underline{M}^{o}(C)\left(z_{3}\right)=(0.3,0.3,0.6),}{\underline{M}^{o}(C)\left(z_{4}\right)=(0.6,0.4,0.4)} \\
\bar{M}^{o}(C)\left(z_{1}\right)=(0.4,0.3,0.5), \bar{M}^{o}(C)\left(z_{2}\right)=(0.4,0.5,0.7), \bar{M}^{o}(C)\left(z_{3}\right)=(0.6,0.3,0.4), \\
\bar{M}^{o}(C)\left(z_{4}\right)=(0.5,0.5,0.5) \\
\underline{M}^{p}(C)\left(z_{1}\right)=(0.3,0.5,0.6),{ }^{p}(C)\left(z_{2}\right)=(0.3,0.5,0.8), \underline{M}^{p}(C)\left(z_{3}\right)=(0.3,0.5,0.9), \\
\bar{M}^{o}(C)\left(z_{1}\right)=(0.6,0.3,0.2), \bar{M}^{o}(C)\left(z_{4}\right)=(0.3,0.5,0.9) \\
\bar{M}^{o}(C)\left(z_{2}\right)=(0.7,0.2,0.5), \bar{M}^{o}(C)\left(z_{3}\right)=(0.7,0.2,0.4)
\end{gathered}
$$


According Definition 4, we have the following:

$$
\begin{gathered}
\left(\underline{M}^{o}(C)+\bar{M}^{o}(C)\right)\left(z_{1}\right)=(0.64,0.15,0.2),\left(\underline{M}^{o}(C)+\bar{M}^{o}(C)\right)\left(z_{2}\right)=(0.7,0.2,0.42), \\
\left(\underline{M}^{o}(C)+\bar{M}^{o}(C)\right)\left(z_{3}\right)=(0.72,0.09,0.24),\left(\underline{M}^{o}(C)+\bar{M}^{o}(C)\right)\left(z_{4}\right)=(0.8,0.2,0.2) \\
\left(\underline{M}^{p}(C)+\bar{M}^{p}(C)\right)\left(z_{1}\right)=(0.72,0.15,0.12),\left(\underline{M}^{p}(C)+\bar{M}^{p}(C)\right)\left(z_{2}\right)=(0.79,0.1,0.4), \\
\left(\underline{M}^{p}(C)+\bar{M}^{p}(C)\right)\left(z_{3}\right)=(0.79,0.1,0.18),\left(\underline{M}^{p}(C)+\bar{M}^{p}(C)\right)\left(z_{4}\right)=(0.79,0.1,0.36)
\end{gathered}
$$

Then, according Definition 5, we have the following:

$$
\begin{aligned}
& S^{o}\left(\alpha_{z_{1}}, \alpha^{*}\right)=0.9315, S^{o}\left(\alpha_{z_{2}}, \alpha^{*}\right)=0.8329, S^{o}\left(\alpha_{z_{3}}, \alpha^{*}\right)=0.8588, S^{o}\left(\alpha_{z_{4}}, \alpha^{*}\right)=0.9428 . \\
& S^{p}\left(\alpha_{z_{1}}, \alpha^{*}\right)=0.9662, S^{p}\left(\alpha_{z_{2}}, \alpha^{*}\right)=0.8865, S^{p}\left(\alpha_{z_{3}}, \alpha^{*}\right)=9677, S^{p}\left(\alpha_{z_{4}}, \alpha^{*}\right)=0.9040 .
\end{aligned}
$$

Then, we have the following:

$$
\begin{gathered}
S^{o}\left(\alpha_{z_{4}}, \alpha^{*}\right)>S^{o}\left(\alpha_{z_{1}}, \alpha^{*}\right)>S^{o}\left(\alpha_{z_{3}}, \alpha^{*}\right)>S^{o}\left(\alpha_{z_{2}}, \alpha^{*}\right) . \\
S^{p}\left(\alpha_{z_{3}}, \alpha^{*}\right)>S^{p}\left(\alpha_{z_{1}}, \alpha^{*}\right)>S^{p}\left(\alpha_{z_{4}}, \alpha^{*}\right)=S^{p}\left(\alpha_{z_{2}}, \alpha^{*}\right) .
\end{gathered}
$$

So, the optimistic optimal choice is to choose $x_{4}$, that is, this given house is "not good" for the customer; the pessimistic optimal choice is to choose $x_{3}$, that is, this given house is "less good" for the customer.

\section{Conclusions}

In this paper, we propose the concept of MGNRS on a single domain and dual domains, and obtain their properties. I addition, we obtain that MGNRS on dual domains will be the same as the MGNRS on a single domain when the two domains are same. Then, we solve a kind of special group decision-making problem (based on neutrosophic relation) using MGNRS on dual domains, and we show the algorithm and give an example to show its feasibility.

In terms of the future direction, we will study other types of combinations of multi-granulation rough sets and neutrosophic sets and obtain their properties. At the same time, exploring the application of MGNRS in totally dependent-neutrosophic sets (see [32]) and related algebraic systems (see [33-35]), and a new aggregation operator, similarity measure, and distance measure (see [36-39]), are also meaningful research directions for the future.

Author Contributions: X.Z. and C.B. initiated the research and wrote the paper, S.S. participated in some of the research work, and F.S. supervised the research work and provided helpful suggestions.

Funding: This research received no external funding.

Acknowledgments: This work was supported by the National Natural Science Foundation of China (Grant No. 61573240, 61473239) and the Graduate Student Innovation Project of Shanghai Maritime University 2017 ycx082.

Conflicts of Interest: The authors declare no conflicts of interest.

\section{References}

1. Chen, H.; Li, T.; Luo, C.; Horng, S.-J.; Wang, G. A decision-theoretic rough set approach for dynamic data mining. IEEE Trans. Fuzzy Syst. 2015, 23, 1958-1970. [CrossRef]

2. Cheng, Y.; Miao, D.Q.; Feng, Q.R. Positive approximation and converse approximation in interval-valued fuzzy rough sets. Inf. Sci. 2011, 181, 2086-2110. [CrossRef]

3. Dai, J.H.; Wang, W.T.; Xu, Q.; Tian, H.W. Uncertainty measurement for interval-valued decision systems based on extended conditional entropy. Knowl.-Based Syst. 2012, 27, 443-450. [CrossRef]

4. Greco, S.; Slowinski, R.; Zielniewicz, P. Putting dominance-based rough set approach and robust ordinal regression together. Decis. Support Syst. 2013, 54, 91-903. [CrossRef] 
5. Jia, X.; Shang, L.; Zhou, B.; Yao, Y. Generalized attribute reduct in rough set theory. Knowl.-Based Syst. 2016, 91, 204-218. [CrossRef]

6. Li, J.H.; Mei, C.L.; Lv, Y.J. Incomplete decision contexts: Approximate concept construction, rule acquisition and knowledge reduction. Int. J. Approx. Reason. 2013, 54, 149-165. [CrossRef]

7. Qian, Y.; Liang, X.; Wang, Q.; Liang, J.; Liu, B.; Skowron, A.; Yao, Y.; Ma, J.; Dang, C. Local rough set: A solution to rough data analysis in big data. Int. J. Approx. Reason. 2018, 97, 38-63. [CrossRef]

8. Zhan, J.; Malik, H.; Akram, M. Novel decision-making algorithms based on intuitionistic fuzzy rough environment. Int. J. Mach. Learn. Cyber. 2018, 1-27. [CrossRef]

9. Smarandache, F. Neutrosophy: Neutrosophic Probability, Set and Logic; American Research Press: Rehoboth, NM, USA, 1998.

10. Smarandache, F. Neutrosophic set-A generialization of the intuitionistics fuzzy sets. Int. J. Pure Appl. Math. 2005, 24, 287-297.

11. Wang, H.; Smarandache, F.; Sunderraman, R.; Zhang, Y.Q. Interval Neutrosophic Sets and Logic: Theory and Applications in Computing; Infinite Study (Cornell University): Ithaca, NY, USA, 2005.

12. Ye, J. A multicriteria decision-making method using aggregation operators for simplified neutrosophic sets. J. Intell. Fuzzy Syst. 2014, 26, 2459-2466.

13. Zhang, X.; Bo, C.; Smarandache, F.; Dai, J. New inclusion relation of neutrosophic sets with applications and related lattice structrue. Int. J. Mach. Learn. Cybern. 2018, 1-11. [CrossRef]

14. Garg, H.; Nancy, G.H. Linguistic single-valued neutrosophic prioritized aggregation operators and their applications to multiple-attribute group decision-making. J. Ambient Intell. Humaniz. Comput. 2018, 1-23. [CrossRef]

15. Garg, H.; Nancy, G.H. Non-linear programming method for multi-criteria decision making problems under interval neutrosophic set environment. Appl. Intell. 2018, 48, 2199-2213. [CrossRef]

16. Nancy, G.H.; Garg, H. Novel single-valued neutrosophic decision making operators under frank norm operations and its application. Int. J. Uncertain. Quantif. 2016, 6, 361-375. [CrossRef]

17. Dubois, D.; Prade, H. Rough fuzzy sets and fuzzy rough sets. Int. J. Gen. Syst. 1990, 17, 191-209. [CrossRef]

18. Broumi, S.; Smarandache, F.; Dhar, M. Rough neutrosophic sets. Neutrosophic Sets Syst. 2014, 3, $62-67$.

19. Yang, H.L.; Zhang, C.L.; Guo, Z.L.; Liu, Y.L.; Liao, X. A hybrid model of single valued neutrosophic sets and rough sets: Single valued neutrosophic rough set model. Soft Comput. 2017, 21, 6253-6267. [CrossRef]

20. Bargiela, A.; Pedrycz, W. Granular computing. In Handbook on Computational Intelligence: Fuzzy Logic, Systems, Artificial Neural Networks, and Learning Systems; World Scientific: Singapore, 2016; Volume 1, pp. 43-66.

21. Qian, Y.; Liang, J.Y.; Yao, Y.Y.; Dang, C.Y. MGRS: A multi-granulation rough set. Inf. Sci. 2010, 180, 949-970. [CrossRef]

22. Skowron, A.; Stepaniuk, J.; Swiniarski, R. Modeling rough granular computing based on approximation spaces. Inf. Sci. 2012, 184, 20-43. [CrossRef]

23. Qian, Y.; Liang, J.Y.; Pedrycz, W.; Dang, C.Y. An efficient accelerator for attribute reduction from incomplete data in rough set framework. Pattern Recognit. 2011, 44, 1658-1670. [CrossRef]

24. AbuDonia, H.M. Multi knowledge based rough approximations and applications. Knowl.-Based Syst. 2012, 26, 20-29. [CrossRef]

25. Wang, H.B.; Smarandache, F.; Zhang, Y.; Sunderraman, R. Single Valued Neutrosophic Sets. Multispace Multistruct. 2010, 4, 410-413.

26. Huang, B.; Guo, C.; Zhuang, Y.; Li, H.; Zhou, X. Intuitionistic fuzzy multi-granulation rough sets. Inf. Sci. 2014, 277, 299-320. [CrossRef]

27. Zhang, X.; Miao, D.; Liu, C.; Le, M. Constructive methods of rough approximation operators and multi-granulation rough sets. Knowl.-Based Syst. 2016, 91, 114-125. [CrossRef]

28. Yao, Y.; She, Y. Rough set models in multi-granulation spaces. Inf. Sci. 2016, 327, 40-56. [CrossRef]

29. Sun, B.; Ma, W.; Qian, Y. Multigranulation fuzzy rough set over two universes and its application to decision making. Knowl.-Based Syst. 2017, 123, 61-74. [CrossRef]

30. Ye, J. Multicriteria decision-making method using the correlation coefficient under single-valued neutrosophic environment. Int. J. Gen. Syst. 2013, 42, 386-394. [CrossRef]

31. Altuzarra, A.; Moreno-Jiménez, J.; Salvador, M. Consensus building in AHP-group decision making: A Bayesian approach. Oper. Res. 2010, 58, 1755-1773. [CrossRef] 
32. Zhang, X.H.; Bo, C.X.; Smarandache, F.; Park, C. New operations of totally dependent-neutrosophic sets and totally dependent-neutrosophic soft sets. Symmetry 2018, 10, 187. [CrossRef]

33. Zhang, X.H.; Smarandache, F.; Liang, X.L. Neutrosophic duplet semi-group and cancellable neutrosophic triplet groups. Symmetry 2017, 9, 275. [CrossRef]

34. Zhang, X.H. Fuzzy anti-grouped filters and fuzzy normal filters in pseudo-BCI algebras. J. Intell. Fuzzy Syst. 2017, 33, 1767-1774. [CrossRef]

35. Zhang, X.H.; Park, C.; Wu, S.P. Soft set theoretical approach to pseudo-BCI algebras. J. Intell. Fuzzy Syst. 2018, 34, 559-568. [CrossRef]

36. Garg, H.; Arora, R. Dual Hesitant Fuzzy soft aggregation operators and their application in decision-making. Cogn. Comput. 2018, 1-21. [CrossRef]

37. Garg, H.; Kumar, K. An advanced study on the similarity measures of intuitionistic fuzzy sets based on the set pair analysis theory and their application in decision making. Soft Comput. 2018, 22, 4959-4970. [CrossRef]

38. Selvachandran, G.; Garg, H.; Quek, S.G. Vague entropy measure for complex vague soft sets. Entropy 2018, 20, 403. [CrossRef]

39. Garg, H. Some new biparametric distance measures on single-valued neutrosophic sets with applications to pattern recognition and medical diagnosis. Information 2017, 8, 162. [CrossRef]

(c) 2018 by the authors. Licensee MDPI, Basel, Switzerland. This article is an open access article distributed under the terms and conditions of the Creative Commons Attribution (CC BY) license (http://creativecommons.org/licenses/by/4.0/). 